\title{
How Dialysis Patients Cope with a Curfew? A Comparison of Psychological Status between Hemodialysis and Peritoneal Dialysis Patients During the COVID-19 Pandemic
}

\author{
Cebrail Karaca $^{\mathrm{a}} \quad$ Necmi Eren $^{\mathrm{b}} \quad$ Mevlut Tamer Dincer $^{\mathrm{a}}$ Senol Turan ${ }^{\mathrm{c}}$ \\ Hatice Kubra Karacad ${ }^{d}$ Mehmet Kucuk ${ }^{e}$ Sennur Kose ${ }^{f}$ Sibel Gokcay Bek ${ }^{b}$

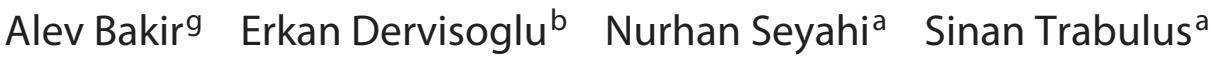 \\ aDepartment of Nephrology, Istanbul University - Cerrahpasa, Cerrahpasa Medical Faculty, Istanbul, Turkey; \\ ${ }^{b}$ Department of Nephrology, Kocaeli University Faculty of Medicine, Kocaeli, Turkey; 'Department of Psychiatry, \\ Istanbul University - Cerrahpasa, Cerrahpasa Medical Faculty, Istanbul, Turkey; ${ }^{d}$ Department of Child and \\ Adolescent Psychiatry, Istanbul Bakirkoy Prof. Dr. Mazhar Osman Training and Research Hospital for Psychiatric \\ and Neurological Diseases, Istanbul, Turkey; ${ }^{e}$ Department of Nephrology, Okmeydani Training and Research \\ Hospital, Istanbul, Turkey; fDepartment of Nephrology, Istanbul Training and Research Hospital, Istanbul, Turkey; \\ 9Department of Biostatistics and Medical Informatics, Halic University, Istanbul, Turkey
}

\section{Keywords}

Anxiety · Depression · COVID-19 · Hemodialysis · Peritoneal dialysis

\begin{abstract}
Introduction: There are many differences between hemodialysis (HD) and peritoneal dialysis (PD) treatments, including their impact on the psychological status of the patients. In this study, our aim was to compare the psychological statuses of HD and PD patients during the social isolation period due to the COVID-19 pandemic. Methods: We conducted this cross-sectional study on adult HD and PD patients when the curfew measures were in effect. We used an electronic form composed of 3 sections to collect data. In the first section, we collected data on the demographics and clinical and laboratory parameters of the patients. The second and third sections consisted of the Hospital Anxiety and Depression Scale (HADS) and the Impact of Event Scale-Revised (IES-R) questionnaires, respectively. Results: The HD $(n=116)$ and PD $(n=130)$ groups were similar regarding age and sex, and
\end{abstract}

they had similar HADS anxiety scores. HADS depression scores were higher in PD patients $(p=0.052)$. IES-R scores were significantly higher in PD patients in comparison to HD patients $(p=0.001)$. Frequencies of abnormal HADS-anxiety $(p=0.035)$ and severe psychological impact $(p=0.001)$ were significantly higher in PD patients. Discussion/Conclusion: During the social isolation period due to the COVID-19 pandemic, HD patients had better mood profiles than PD patients. A more stable daily routine, an uninterrupted face-toface contact with health-care workers, and social support among patients in the in-center dialysis environment might be the cause of the favorable mood status. PD patients might need additional psychological support during those periods.

(c) 2021 S. Karger AG, Basel

\section{Introduction}

On March 11, 2020, the World Health Organization announced the pandemic status for a new type of coronavirus (severe acute respiratory syndrome coronavirus 2) 
infection (COVID-19). The outbreak emerged from $\mathrm{Wu}-$ han city in China [1].

In Turkey, the first PCR-confirmed case was identified on March 11. Since then, a plethora of regulations from the government has come into effect. Schools and all public gathering places, such as cafes, gyms, internet cafes, movie theaters, restaurants, dining places, and patisseries were closed. A total lockdown was declared for people who were over 65 or below 20 years of age or those with a chronic illness. Finally, on April 10, 2020, a total lockdown during weekends was imposed in 30 metropolitan provinces.

The rapid spread of the COVID-19 pandemic all over the world has had a massive impact on health-care systems and therefore on individuals. Studies investigating the short-term mental health effects of the COVID-19 pandemic revealed the harmful psychological consequences of the outbreak on different populations [2]. Still, the mental health effects of the COVID-19 outbreak on patients with chronic medical illnesses remain largely unaddressed.

There are many factors that may lead to a mental disorder, including depression or anxiety among patients undergoing hemodialysis (HD) or peritoneal dialysis (PD) [3]. Apart from disease-related issues such as pain, sleep disturbances, fatigue, uremic symptoms, and restrictions of diet and social life, certain events may aggravate mental disorder in these specific populations. Different psychological measures can be used to evaluate individuals for a range of mental health conditions such as anxiety, depression, and post-traumatic stress disorder. In this study, we aimed to compare the effects of the pandemic and its control measures on the psychological statuses of HD and PD patients.

\section{Materials and Methods}

\section{Study Design}

We performed a cross-sectional study and collected information via electronic survey about the demographics (age, gender, marital status, having children, level of education, household size, and presence of a household member $>65$ years of age), clinical and laboratory data, and psychological statuses of the participants. Using the survey, we tried to find out whether the patients considered the outbreak "very serious," "serious," or "not serious," whether they followed the advices to stay home or felt obliged to leave home for work, and whether they thought they received adequate medical support during the pandemic period.

The study was conducted between May 11 and May 31, 2020, among patients attending $3 \mathrm{HD}$ (in Istanbul province) and $2 \mathrm{PD}$ centers (Istanbul and Kocaeli provinces), located in the Marmara Region and who were under the same administrative regulations regarding pandemic-related restrictions.

\section{Participants}

Only adult patients ( $>18$ years of age) were eligible to participate in this study. The first group of participants consisted of HD patients who were under regular follow-up in 3 different HD centers (one university hospital and 2 private centers) and the second group included PD patients from 2 PD centers (one university and one training and research hospital). A total of 246 individuals were eligible for the study. The Hospital Anxiety and Depression Scale (HADS) and the Impact of Event Scale-Revised (IES-R) questionnaires were completely filled by the $116 \mathrm{HD}$ and $130 \mathrm{PD}$ patients. We evaluated the presence of the following comorbid diseases: diabetes mellitus, hypertension, asthma, chronic obstructive pulmonary disease, coronary arterial disease, and heart failure.

\section{Survey and Data Collection}

We used an electronic survey created using the Google Forms platform. A Google account was set up (https://forms. gle/4Em91L7J5LZRuNCRA) and managed by the lead researcher to collect the survey responses, which were recorded on the designed platform. The survey consisted of 3 sections. In the first section, demographics and the clinical and social data of the patients were collected. The patients were also asked questions to find out how many times they had left their house and had undergone HD. In the second section, an electronic version of the HADS was used. And finally, in the last section, the IES-R questionnaire was used $[4,5]$. HD patients filled out the forms using smartphones during their HD sessions, while PD patients filled out the survey using smartphones or computers via a Web link sent to their phones.

\section{Hospital Anxiety Depression Scale}

The HADS is commonly used to assess the anxiety and depression of patients [4]. The term "hospital" in its title suggests that it is used for patients only, but many studies have confirmed that it can be used in community settings and primary care medical practice too [6]. The HADS contains 2 subscales that measure symptoms of depression (HADS-D; 7 items) and anxiety (HADS-A; 7 items) during the previous week. The items are scored on a fourpoint rating scale from 0 to 3 , and for each subscale, the total score is at most 21 . We used the validated Turkish version of the HADS in our study. The HADS scores can be interpreted using cutoff values. For the Turkish version, a cutoff value of 10 for the HADS-A score and a cutoff value of 7 for the HADS-D score was proposed to classify the scores as "abnormal" [7].

\section{Impact of Event Scale-Revised}

The IES-R tool is commonly used to determine the severity of post-traumatic stress disorder [5] and has been used to measure stress after the COVID-19 pandemic [8]. The scale consists of 22 items, and each item is rated on a five-point rating scale from 0 to 4. The scale is composed of 3 subscales and aims at measuring the mean avoidance, intrusion, and hyperarousal [9]. Its validity and reliability in Turkey were demonstrated by Corapcioglu et al. [10] in 2006, and we used this validated Turkish version. The Turkish version of the IES-R is validated with a good diagnostic performance and a high internal consistency for the cutoff points between 24 and 33. A total IES-R score above 33 suggests the presence of post-traumatic stress disorder. 
Table 1. Demographic, clinical, and sociocultural data of the study participants

\begin{tabular}{|c|c|c|c|}
\hline & $\mathrm{HD}(n=116)$ & $\mathrm{PD}(n=130)$ & $p$ value \\
\hline Age $($ mean $+S D)$, min-max, years & $59.6 \pm 14.3(19-88)$ & $56.8 \pm 14.3(19-88)$ & 0.193 \\
\hline Gender (male, \%) & 59.5 & 49.2 & 0.107 \\
\hline Comorbid disease, $\%$ & 55.2 & 76.2 & 0.001 \\
\hline Diabetes mellitus, \% & 30.2 & 33.8 & 0.538 \\
\hline Hypertension, $\%$ & 35.3 & 65.4 & $<0.001$ \\
\hline Time on dialysis, mean $\pm \mathrm{SD}$, years & $6.3 \pm 6.1$ & $5.6 \pm 4.0$ & 0.523 \\
\hline Residual renal function, \% & NA & 63.8 & NA \\
\hline Marital status (married, \%) & 60.3 & 81.5 & $<0.001$ \\
\hline Have children (yes, \%) & 80.2 & 93.1 & $<0.001$ \\
\hline \multicolumn{4}{|l|}{ Level of education, $\%$} \\
\hline Primary school & 75.0 & 71.6 & \\
\hline \multicolumn{4}{|l|}{ Household size, $\%$} \\
\hline $4-6$ & 25.9 & 44.6 & 0.008 \\
\hline$>6$ & 2.6 & 1.5 & \\
\hline Presence of a household member $>65$ years of age (yes, \%) & 41.4 & 31.5 & 0.109 \\
\hline \multicolumn{4}{|l|}{ Considering the outbreak, $\%$} \\
\hline Very serious & 62.9 & 70.0 & 0.441 \\
\hline Serious & 35.3 & 29.2 & \\
\hline Not serious & 1.7 & 0.8 & \\
\hline Following the advices to stay home (yes, \%) & 99.1 & 97.7 & 0.624 \\
\hline Feeling obliged to leave home for work (yes, \%) & 16.4 & 7.7 & 0.056 \\
\hline $\begin{array}{l}\text { Thinking that they received adequate medical support during the } \\
\text { pandemic period (yes, \%) }\end{array}$ & 81.0 & 75.4 & 0.285 \\
\hline
\end{tabular}

NA, not available; SD, standard deviation; HD, hemodialysis; PD, peritoneal dialysis. Significant $p$ values are written in bold.

\section{Statistical Analysis}

Descriptive statistics were expressed as mean, standard deviation, median, minimum, and maximum for continuous data and as count and proportion for categorical data. The categorical data were analyzed using the $\chi^{2}$ or Fisher's exact tests. The distribution normality of the continuous variables was calculated with the Shapiro-Wilk test. Normally distributed continuous variables were compared using the independent samples $t$ test, while the MannWhitney $U$ test was used for the non-normally distributed data. Statistical analyses were performed using IBM SPSS for Windows v.24 software and were reported with $95 \%$ confidence intervals. Values of $p<0.05$ were considered significant.

\section{Results}

\section{Participants, Demographic, Clinical, and Sociocultural Data}

The demographic, clinical, and sociocultural data of the participants are shown in Table 1 and online suppl. Table 1; for all online suppl. material, see www.karger. com/doi/10.1159/000517839. Age and gender distribution among the $\mathrm{HD}$ and $\mathrm{PD}$ patients were similar. The prevalence of diabetes mellitus was similar between the 2 groups; however, hypertension was more common among PD patients. Regarding the sociocultural status, marriage and having a child was more common among $\mathrm{PD}$ patients, and they lived with a higher number of persons in the same house. However, the educational status, the presence of a household member $>65$ years of age, consideration of the seriousness of the outbreak, following the advices to stay home, the obligation of leaving home for work, and the perception of having adequate medical support were similar between $\mathrm{HD}$ and $\mathrm{PD}$ patients (Table 1).

Patients with HD and PD reported that they left the house for $3.6 \pm 1.3$ times/week and $1.1 \pm 1.4$ times/week on average, respectively $(p<0.001)$. Most $(93.9 \%)$ of the HD patients were receiving dialysis 3 times per week. Vascular access through arteriovenous fistulas was the 
Table 2. The HADS and IES-R scores of the study groups

\begin{tabular}{lccc}
\hline & $\mathrm{HD}^{*}(n=116)$ & $\mathrm{PD}^{*}(n=130)$ & $p$ value \\
\hline HADS-A score $($ normal $<7)$ & $6.6 \pm 4.0(6.0)[0-20]$ & $7.5 \pm 4.5(7.0)[0-18]$ & 0.098 \\
HADS-D score $($ normal $<10)$ & $6.8 \pm 4.0(6.5)[0-19]$ & $7.9 \pm 4.4(7.0)[0-20]$ & 0.052 \\
IES-R score (normal <33) & $24.0 \pm 12.5(24.0)[0-66]$ & $29.8 \pm 13.8(28.0)[2-68]$ & $\mathbf{0 . 0 0 1}$ \\
\hline
\end{tabular}

HADS, Hospital Anxiety and Depression Scale; IES-R, Impact of Event Scale-Revised; SD, standard deviation; HD, hemodialysis; PD, peritoneal dialysis; A, anxiety; D, depression. Significant $p$ values are written in bold. * Data are expressed as mean $\pm \mathrm{SD}$ (median) [range].

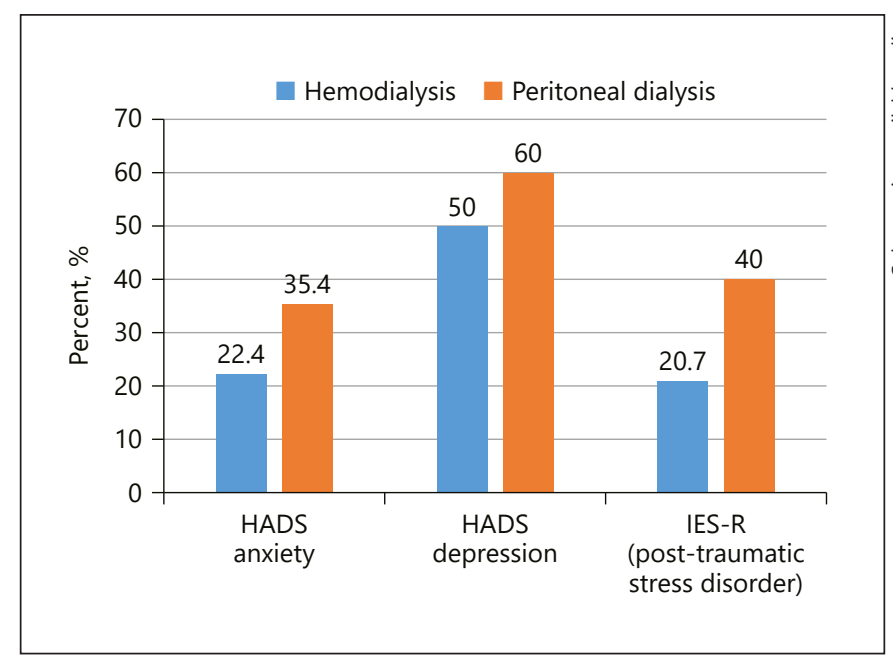

Fig. 1. The percentage of patients classified as abnormal according to the HADS-A and HADS-D scores and the percentage of patients with post-traumatic stress disorder according to IES-R scores for each study group (HADS-A [ $p=0.035]$, HADS-D $[p=0.125]$, and IES-R $[p=0.001])$. HADS, Hospital Anxiety and Depression Scale; A, anxiety; D, depression; IES-R, Impact of Event Scale-Revised.

most frequently used method among HD patients. Continuous ambulatory PD was more common compared to automated PD (79.2\% vs. $20.8 \%, p<0.001)$ among PD patients. Dialysis adequacy, expressed as a Kt/V $>1.2$ for $\mathrm{HD}$ patients, and a weekly $\mathrm{Kt} / \mathrm{V} \geq 1.7$ for $\mathrm{PD}$ patients was observed to be 93.3 and 82.9 for HD and PD patients, respectively $(p=0.015)$ (Table 1$)$. The mean hemoglobin level was $11.3 \pm 1.5 \mathrm{~g} / \mathrm{dL}$ among HD patients and $10.7 \pm$ $1.5 \mathrm{~g} / \mathrm{dL}$ among PD patients $(p=0.004)$.

\section{HADS and IES-R Results}

Table 2 shows the HADS and IES-R scores. Both groups exhibited similar HADS-A scores. HADS-D scores were higher in PD patients; however, the statistical significance was borderline $(p=0.052)$. Additionally, the
IES-R scores were significantly higher in $\mathrm{PD}$ patients than those in HD patients $(p=0.001)$.

The percentage of patients classified as abnormal according to the HADS-A and HADS-D scores and the percentage of patients with post-traumatic stress disorder according to the IES-R scores for each study group is shown in Figure 1. Frequencies of abnormal HADS-A score ( $p=$ $0.035)$ and severe psychological impact $(p=0.001)$ were significantly higher in $\mathrm{PD}$ patients in comparison to HD patients. The frequency of abnormal HADS-D scores was similar between the 2 groups $(p=0.125)$.

\section{Discussion}

To the best of our knowledge, this is the first study that compares the impact of social distancing regulations during the COVID-19 pandemic on the mental status of HD and PD patients. We found that pandemic control measures had a greater impact on the mental statuses of PD patients.

Several studies have compared the mental status of HD and PD patients during the pre-pandemic period [1118]. While some studies have not found a significant difference between the 2 dialysis methods in terms of mental status $[11,12]$, some studies suggested that the mental statuses of PD patients were superior [13-16], while others favored the mental statuses of HD patients [17, 18].

One of the studies that have shown that there is no significant difference between the mental statuses of PD and HD patients is that of Stasiak et al. [11]. The researchers studied $128 \mathrm{HD}$ and 27 PD patients using the Beck Depression Inventory, the Beck Anxiety Inventory, and HADS and concluded that the type of dialysis performed did not influence the prevalence of anxiety and depression in chronic kidney disease patients [11]. In a prospective cohort study, Wu et al. [12] examined $698 \mathrm{HD}$ and 230 PD patients and compared the self-reported health- 
related quality of life and overall health status of HD and PD patients at the initiation of dialysis therapy and 1 year later. The authors concluded that PD patients did not have a better quality of life than the HD patients who were begun renal replacement therapy. In general, HD patients may have maintained their general health status to a greater extent than the PD patients.

There are studies in the literature which suggest that PD patients are better than HD patients in terms of mental status. Ginieri-Coccossis et al. [13] investigated 77 incenter $\mathrm{HD}$ and 58 continuous ambulatory PD patients using the World Health Organization Quality of Life Questionnaire, the General Health Questionnaire, and the Multidimensional Health Locus of Control and concluded that HD patients experienced more depression symptoms than PD patients. In a seminal study, Kimmel et al. [16] examined all the Medicare-enrolled dialysis patients who were hospitalized in the United States during $1993(n=176,368)$ and found that the adjusted risk of hospitalization for PD patients was lower than that of $\mathrm{HD}$ patients for any mental disorder, depression, and alcohol and drug use.

Contrary to that, in some studies, HD patients were shown to be better than PD patients in terms of mental status. For instance, Griva et al. [17] compared the quality of life and emotional adjustment between patients on home-based PD $(n=201)$ regimens and patients on community HD programs $(n=232)$ using the HADS and Kidney Disease Quality of Life Short Form. The authors concluded that PD patients reported higher symptoms of depression and lower physical health yet a higher satisfaction with the care given. Lin et al. [18] compared the prevalence of depression using the Taiwanese Depression Questionnaire among HD, PD, and transplantation patients and found that the prevalence of depression among PD patients was higher than those among HD and transplant patients. We also found that PD patients had a higher rate of abnormal HADS-A and IES-R scores. Additionally, although of borderline significance, we noticed that PD patients had higher HADS-D scores; this finding is in line with the study of Griva et al. [17].

In-center HD is a socialized environment that has its own advantages and pitfalls. Patients are more dependent on health-care facilities, and they need to be mobile in order to reach the dialysis centers. The dialysis units may foster social support among patients' having issues related to emotional contagion; witnessing adverse outcomes in others may trigger distress. Additionally, distress in HD patients may be related to procedural aspects of treatment such as the need to travel to dialysis centers, trans- portation issues, spending considerable time in medical environments (i.e., dialysis wards), and the prolonged sitting time during dialysis. Distress may also be related to the fear of access complications, dialysis-related symptoms, and side effects, which are common concerns among HD patients. Some dialysis centers where COVID-19 patients are also accepted may leave the patients prone to a more stressful environment. The advantages of spending time in the health-care facilities for HD patients could be seeing the health-care professionals on a regular basis and being able to seek first-hand answers from them about the pandemic. According to our results, the advantages of in-center HD were more prominent than pitfalls in a setting of social isolation due to the pandemic.

On the other hand, PD patients are less dependent on health-care settings, and they may isolate themselves at home without an interruption during their renal replacement therapy. However, PD patients did not leave their homes, and they did not have regular face-to-face contacts with health-care professionals during the pandemic, in contrast to HD patients. Additionally, daily life routines were changed more profoundly for PD patients in comparison to HD patients. PD patients who were used to having a more flexible daily routine were forced to stay home, while HD patients continued their regular visits to HD centers. Worse IES-R scores in PD patients might also reflect this traumatic experience.

We used the translated and validated version of psychological assessment tools. Translating psychological assessment tools from one language to another is not just a matter of finding the right words; it should involve, in addition to literal translation, a process of revision, modification, and amendment of the content of those tools to fit the receiving culture [19]. Translated versions of the psychological tests must be statistically validated before being used. During statistical validation, the receiver-operating curve analysis was used, and different cutoff values were found for the Turkish version of the HADS [7].

There were some limitations to our study. Due to its cross-sectional nature, it was hard to infer the causality in the study. We measured the compliance with the curfew using one self-reported questionnaire; therefore, an information bias should be considered. Our study was performed in 2 cities; however, presence of a sociodemographic difference between those 2 cities is unlikely since they are neighboring cities and had the same regulations during the pandemic.

In conclusion, our study might be considered unique since it was performed during the COVID-19 pandemic; a period with unprecedented social isolation measures. 
According to our findings, during this period, HD patients had better mood profiles than PD patients. We can argue that face-to-face contact with health-care workers and social support among patients in the in-center dialysis environment might be the cause of the more favorable mood status in HD patients than PD patients. PD patients might need additional psychological support during such periods.

\section{Acknowledgements}

The authors thank Sengul Gencoglu, Suna Kalender, and Rukiye Dolgun for their help during the data collection.

\section{Statement of Ethics}

The study was approved by the Ministry of Health (2020-0514T14_08_10) and by the local medical Ethics Committee (approval no: 83088843-604). Informed consent was obtained from all individual participants included in the study.

\section{Conflict of Interest Statement}

The authors have declared that no competing interests exist.

\section{Funding Sources}

The authors received no specific funding for this work.

\section{Author Contributions}

The individual contribution of each co-author is listed below using the following keys: 1 - Conception or design, or analysis and interpretation of data, or both; 2 - Drafting the article or revising it; 3 - Providing intellectual content of critical importance to the work described; and 4 - final approval of the version to be published.

Cebrail Karaca: 1, 2, 3, and 4. Necmi Eren: 1, 2, and 3. Mevlut Tamer Dincer: 2 and 4. Senol Turan: 1 and 4. Hatice Kubra Karaca: 1 and 4. Mehmet Kucuk: 1 and 4. Sennur Kose: 1 and 4. Sibel Gokcay Bek: 1 and 4. Alev Bakir: 1 and 2. Erkan Dervisoglu: 1 and 3. Nurhan Seyahi: 1, 2, 3, and 4. Sinan Trabulus: 1, 2, 3, and 4.

\section{References}

1 Huang C, Wang Y, Li X, Ren L, Zhao J, Hu Y, et al. Clinical features of patients infected with 2019 novel coronavirus in Wuhan, China. Lancet. 2020;395(10223):497-506.

2 Vindegaard N, Benros ME. COVID-19 pandemic and mental health consequences: systematic review of the current evidence. Brain Behav Immun. 2020;89:531-42.

3 Usher K, Bhullar N, Jackson D. Life in the pandemic: social isolation and mental health. J Clin Nurs. 2020;29(15-16):2756-7.

4 Zigmond AS, Snaith RP. The hospital anxiety and depression scale. Acta Psychiatr Scand. 1983;67(6):361-70.

5 Weiss DS, Marmar CR. The impact of event scale: revised. In: Wilson JP, Keane TM, editors. Assessing psychological trauma and PTSD. The Guilford Press; 1997. p. 399-411.

6 Snaith RP. The hospital anxiety and depression scale. Health Qual Life Outcomes. 2003; 1:29.

7 Aydemir O, Guvenir T, Kuey L, Kultur S. Validity and reliability of Turkish version of hospital anxiety and depression scale. Turkish J Psychiatry. 1997;8(4):280-7.
8 Wang C, Pan R, Wan X, Tan Y, Xu L, Ho CS, et al. Immediate psychological responses and associated factors during the initial stage of the 2019 coronavirus disease (COVID-19) epidemic among the general population in China. Int J Environ Res Public Health. 2020; 17(5):1729.

9 Creamer M, Bell R, Failla S. Psychometric properties of the impact of event scale: revised. Behav Res Ther. 2003;41(12):1489-96.

10 Corapcioglu A, Yargic I, Geyran P, Kocabasoglu N. Validity and reliability of Turkish version of "Impact of Event Scale-Revised" (IESR). New Symposium J. 2006;44(1):14-22.

11 Stasiak CE, Bazan KS, Kuss RS, Schuinski AF, Baroni G. Prevalence of anxiety and depression and its comorbidities in patients with chronic kidney disease on hemodialysis and peritoneal dialysis. J Bras Nefrol. 2014;36(3): 325-31.

$12 \mathrm{Wu}$ AW, Fink NE, Marsh-Manzi JV, Meyer $\mathrm{KB}$, Finkelstein FO, Chapman MM, et al. Changes in quality of life during hemodialysis and peritoneal dialysis treatment: generic and disease specific measures. J Am Soc Nephrol. 2004;15(3):743-53.

13 Ginieri-Coccossis M, Theofilou P, Synodinou C, Tomaras V, Soldatos C. Quality of life, mental health and health beliefs in haemodialysis and peritoneal dialysis patients: investigating differences in early and later years of current treatment. BMC Nephrol. 2008;9:14.
14 Juergensen E, Wuerth D, Finkelstein SH, Juergensen PH, Bekui A, Finkelstein FO. Hemodialysis and peritoneal dialysis: patients' assessment of their satisfaction with therapy and the impact of the therapy on their lives. Clin J Am Soc Nephrol. 2006; 1(6):1191-6.

15 Theofilou P. Quality of life in patients undergoing hemodialysis or peritoneal dialysis treatment. J Clin Med Res. 2011;3(3):132-8.

16 Kimmel PL, Thamer M, Richard CM, Ray NF. Psychiatric illness in patients with end-stage renal disease. Am J Med. 1998;105(3):214-21.

17 Griva K, Kang AW, Yu ZL, Mooppil NK, Foo $\mathrm{M}$, Chan CM, et al. Quality of life and emotional distress between patients on peritoneal dialysis versus community-based hemodialysis. Qual Life Res. 2014;23(1):57-66.

18 Lin Y-H, Yang Y, Chen S-Y, Chang C-C, Chiu $\mathrm{P}-\mathrm{F}$, Huang C-Y. The depression status of patients with end-stage renal disease in different renal replacement therapies. Int J Urol Nurs. 2011;5(1):14-20.

19 Selvi Y, Gulec M, Aydin A, Besiroglu L. Psychometric evaluation of the Turkish language version of the Profile of Mood States (POMS). J Mood Disord. 2011;1(4):152-61. 